\title{
The influence of new NLTE model atmospheres with wind effects in nebular modeling
}

\author{
T.M. Yamamoto ${ }^{1,2}$, F. H. Sellmaier ${ }^{2}$, A. W. A. Pauldrach ${ }^{2}$ and T. Hoffmann ${ }^{2}$ \\ ${ }^{1}$ Institute of Astronomy, The University of Tokyo, Mitaka, Tokyo, 181 Japan; \\ ${ }^{2}$ Institut für Astronomie und Astrophysik der Universität München, München, Germany
}

Emergent fluxes from a non-LTE model atmosphere code designed to calculate realistic $\mathrm{O}$ star atmospheres are applied to nebular calculations. The stellar models include wind and blocking effects due to several million doppler-shifted lines in the radiation driven atmosphere in a full NLTE treatment. These lines, which arise from heavy elements, reduce the stellar flux drastically between 228 and $912 \AA$ (Pauldrach et al. 1994) - the most important frequency regime for ionization in gaseous nebulae. We have three models with effective temperatures of 50000,40000 and $35000 \mathrm{~K}$ and $\log g$ values of $3.9,3.8$ and 3.5 respectively. They have been used to model HII regions and their use has led to significant differences in the ionization structure of the nebular models.

There are two important changes in the emergent flux of the NLTE atmosphere when compared with standard LTE atmospheres. The flux between 350 and $540 \AA$ is reduced in all models, decreasing the degree of ionization for $\mathrm{S}^{++}$and $\mathrm{N}^{++}$. On the other hand, the flux between 228 and $350 \AA$ is enhanced drastically for lower $T_{\text {eff }}$, increasing the ionization fractions of $\mathrm{O}^{++}$and $\mathrm{Ne}^{++}$significantly. In the case of $35000 \mathrm{~K}$, the number of ionizing photons for $\mathrm{Ne}^{++}$has been increased by a factor of 100 , leading to a solution of the problem that previous nebular models using the heretofore available fluxes were not able to reproduce the high degree of ionization of $\mathrm{Ne}^{++}$derived from observations of HII regions (Sellmaier et al. 1996). The agreement of calculated and observed line strengths of the doubly ionized elements in HII regions with $T_{\text {eff }}$ below $50000 \mathrm{~K}$ means that model calculations of PNe with cool central stars should also be performed with NLTE model atmospheres.

For O stars it is vital to include the blocking effects of metal lines but for central stars of planetary nebulae (CSPNe) there has been no investigation. Whether or not the blocking effects of metal lines are important for CSPNe is uncertain. However, stellar wind-effects and NLTE are an inevitable part of a hot atmosphere. Another consequence, which has not been discussed above, are shocks due to accelerated particles in the outer part of stellar atmospheres. The NLTE models have included this effect in a spherical extended atmosphere and show a significant rise in the flux shortwards of $228 \AA$, increasing the ionization fraction of $\mathrm{N}^{4+}, \mathrm{O}^{3+}, \mathrm{Ne}^{3+}$ and other high ionization stages. For higher temperatures a non-negligible rise of the EUV spectrum can be expected. Thus a quantitative understanding of PNe will depend on the modeling of these effects.

\section{REFERENCES}

Pauldrach, A. W. A., Kudritzki, R. P., Puls, J., Butler, K., Hunsinger, J., 1994, Astron. Astrophys., 283, 525.

Sellmaier, F. H., Yamamoto, T., Pauldrach, A. W. A., Rubin, R. H., 1996, Astron. Astrophys., 305, L37.

(T. Yamamoto in the letter is the same as the first author T. M. Yamamoto) 